\title{
The Influence of Geometric Characteristics on Energy Absorption of M-Type Folded Core
}

\author{
Zhou Huazhi, a, Wang Zhijin ${ }^{1, b}$ \\ ${ }^{1}$ College of Aerospace Engineering, Nanjing University of Aeronautics and Astronautics, Nanjing \\ 210016, China \\ a2543436974@qq.com, b'zhijin@nuaa.edu.cn
}

\begin{abstract}
Keywords: Folded Core; Geometric Characteristics; Anti-crash; Energy Absorption Character;
\end{abstract} Finite element method.

\begin{abstract}
The sandwich plate with folded core is a new type of sandwich structure. It has good design ability and has gained interest in the aerospace industry as a promising sandwich core structure. In this paper, energy absorption characters of M-type folded core are studied. Finite element models are set up for folded cores with different geometric parameters. The calculation result of the finite element model agrees well with the experiment data. And the energy absorption coefficient of folded core is 2.6 times as much as the coefficient of honeycomb core with the same height and density. Besides, influences of each geometric parameter on energy absorption characters are analyzed. And some useful results have been obtained. These results can be helpful when designing folded core as anti-crash structure.
\end{abstract}

\section{Introduction}

Honeycomb core sandwich structures have a successful application as lightweight structures in aircraft design due to their superior stiffness-weight ratio and strength-weight ratio. However, the applications of honeycomb core sandwich structures are limited due to their accumulation of humidity, the complexity in manufacturing and the vulnerability against impact loads. In order to obtain new sandwich structures, the transnational project CELPACT (Cellular Structures for Impact Performance) has been set up and completed ${ }^{[1]}$. The project concentrated on advanced cellular core materials for potential applications in future primary aircraft structures with lower fabrication costs and improved impact performance. The folded core sandwich structures are one of their achievements. The folded core structures, as shown in Fig 1, are geometric structures formed by folding plates or foils according to regular repeated lines. According to different lines, M-type(Fig 1(a),(b)), V-type and other types of folded core structures can be formed, even shells with large curvature (Fig 1(c)), stepped plate(Fig 1(d)) and plates with variable thickness(Fig 1(e)) can be manufactured using folded core. Besides, folded core sandwich structures would not accumulate humidity, and have advantages of normal sandwich structures as high stiffness-weight ratio. Therefore, folded core sandwich structures have a potential application value in aircraft industry.

Some studies have been carried out on folded core. The sound insulation performance, the thermal protective performance, the radar cross section(RCS) and the mechanics model of folded core have been studied through experiments and simulations by Wang Zhijin and Xu Qinghua ${ }^{[2-7]}$. It has been proved that sandwich structures with folded core have good performance in all the areas above. The electromagnetic shielding performance of folded core has been studied by Shao Changlin ${ }^{[8]}$. The electromagnetic shielding performance improves efficiency with the help of folded core from wire mesh. The sound insulation performance has also been studied ${ }^{[9]}$, and some useful results have been achieved. Many experiments and simulations have also been conducted on the folded core by S.Heimbs ${ }^{[10-18]}$. The impact properties of folded core from CF/EP(carbon fiber/epoxy) have been studied. Compression and shear experiments have been conducted on folded core from CFRP(carbon fiber-reinforced plastic) and aramid paper. Besides, a numerical simulation method has been carried out to study the mechanical properties in all period from starting loading to the densification of the core. Different methods were used to model imperfections of folded cores. Compression, shear and 
impact properties of different folded core samples were analyzed using the numerical simulation method. A series of experiments have been conducted on the mechanical properties of aramid paper (a base material of folded core) by Sebastian Fischer ${ }^{[19]}$. And the tensile and compression stress-strain curves have been achieved. The residual strength and the impact property of honeycomb core and folded core sandwich structures have been studied with the help of experiments and numerical simulations. The stiffness and failure behavior of folded core sandwich structure under combined transverse shear and compression have also been studied. In order to model folded core with higher accuracy, a new way to model the material imperfections and geometrical defects of folded core has been set up by E. Baranger, P. A. Guidault and C. Cluzel ${ }^{[20-21]}$. The issue of the relation between the strength of wedge-shape folded core sandwich structures and the geometrical parameters has been considered by Falk Hähnel and Klaus Wolf ${ }^{[22]}$.

Folded core sandwich structures have good design ability, so structures can be manufactured to meet the needs of anti-crash structures. In this paper, the Energy Absorption Coefficient(EAC) of folded core is studied using FEM(the finite element method), and it is compared with the result of honeycomb core ${ }^{[23]}$, the folded core's EAC is 2.6 times as much as the EAC of honeycomb core. Besides, the influence of geometric parameters on EAC is analyzed.

\section{M-type folded core}

M-type folded core. M-type folded core with constant height is composed of parallelogram element 1 and rectangular element 2. The folded core is formed from folding of foil according to folding lines. The folding lines and geometrical parameters of folded core are shown in Fig 2 . Line 3 is M-shape line, line 4 is saw-tooth line.

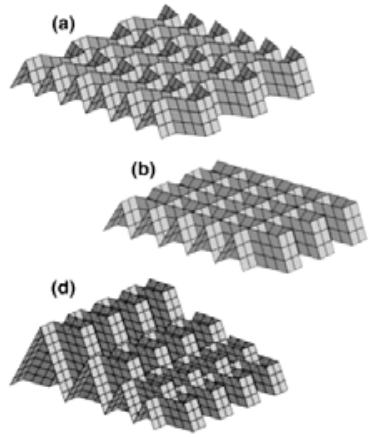

Fig.1 Some kinds of folded core

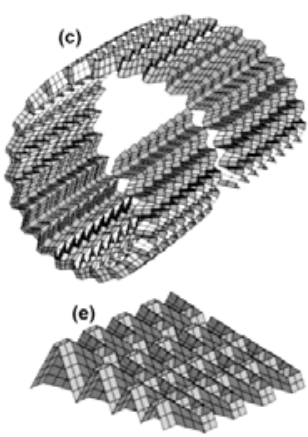

Fig.2 M-type folded core and its folding lines

The relation of geometrical parameters of folded core and parameters of folding lines can been expressed as:

$$
\begin{aligned}
& L_{0}=H / \cos (\alpha) \\
& V_{0}=A \cos (\lambda) \sin (\alpha) \\
& S_{0}=B+A \sqrt{1-\cos ^{2}(\lambda) \sin ^{2}(\alpha)} \\
& B=B
\end{aligned} .
$$

In formula (1):A-the top side length of parallelogram element;2 $\alpha$-the angle of saw-tooth lines; $\lambda+90^{\circ}$-the angle of $M$-shape lines; $B$-he interval of saw-tooth lines; $H$-the height of folded core; $2 L_{0}$-the vertical step length of folding lines; $V_{0}$-the folded amplitude of $M$-shape lines of fold lines; $2 S_{0}$-the step length of $\mathrm{M}$-shape lines of folding lines.

The mass of one folded core cell can be expressed as:

$m_{\text {cell }}=4 * H / \cos (\alpha) *(A \sin (\lambda)+B) * t * \rho$

In formula (2), $t$-the thickness of the foil; $\rho$-the density of the foil.

Generally, EAC is used to measure the energy absorption ability of a structure. EAC is defined as the energy absorbed by per unit mass of anti-crash structure:

$\mathrm{EAC}=E / m$ 
The energy is absorbed through deformation and failure of the structure. The absorbed energy $E$ is equal to the integral area of the force-displacement curve. And $m$ is the mass of the folded core.

Finite element model of M-type folded core. The sandwich plates with folded core consist of two panels and a folded core, which is placed in the middle of the two panels. In finite element models, the panels can be simply seen as rigid shells. It is because that the panels absorb little energy during the compression period.

The folded core is made from aramid paper. The complex stress-strain curve of aramid paper is simplified as shown in Fig 3. The original stress-strain curve and its simplification are compared. The original curve can be achieved from reference paper [10]. Because of little absorbed energy during the elastic process, the simplification of the elastic property of the aramid paper is acceptable. The simulation result also proves it.

A parametric modeling method using Python and ABAQUS is used to model the folded core. The cell number of the model is set as $3 * 6$ cells. In order to reduce calculation cost meanwhile keeping the calculation accurate, the element's size is optimized to be $1 \mathrm{~mm}$. The element type is set as S4R.

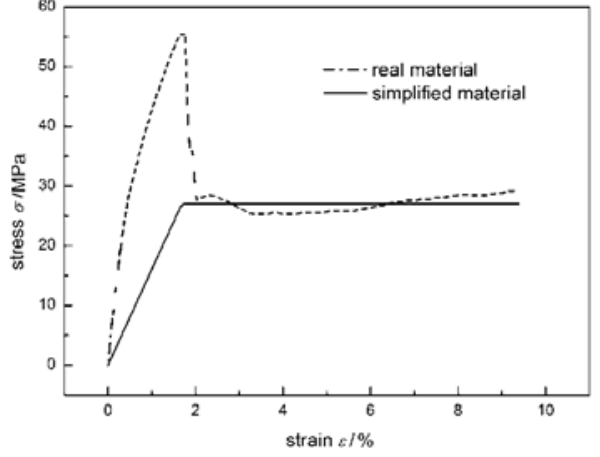

Fig.3 The stress-strain curve of material

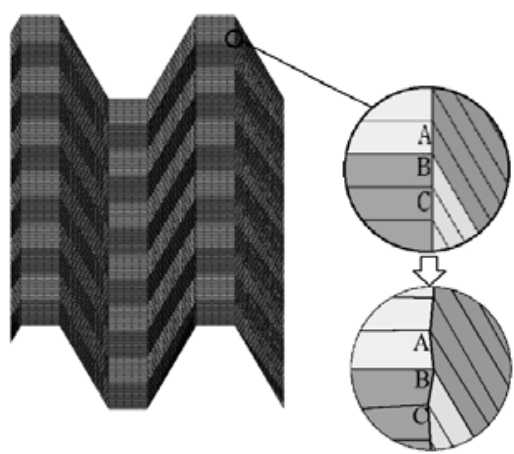

Fig.4 Node shaking Fig.5 The finite element model of the

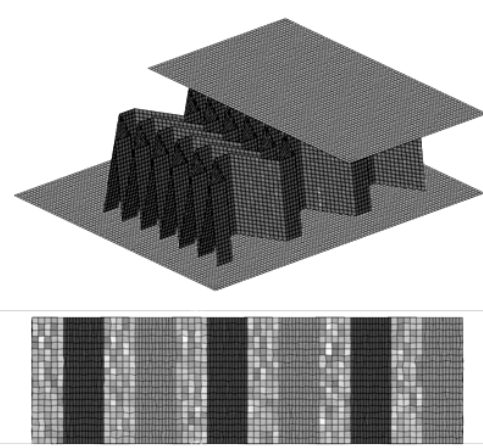

the folded core sandwich structure

In the manufacturing process of folded core, imperfections can not be avoided. In this paper, the node shaking method is used to model the imperfections. It means that each vertex of the finite element model is positioned randomly near its nominal position. The node shaking method is shown in Fig 4. It is effective to simulate the imperfections of folded core generated during the manufacturing process.

The panels and the core are connected using the contact constraint. The contact algorithm is the penalty method. The friction coefficient is set as infinite to simulate the adhesive bounding between the folded core and the panels. And self-contact is definite due to the huge deformation during the analysis. In self-contact, the friction coefficient is set as 0 .

The final model can be seen as Fig 5 .

\section{Analysis result.}

Typical failure behavior of folded core under compression. The typical $\sigma_{\mathrm{e}}-\varepsilon_{\mathrm{e}}$ curve is shown in Fig 7. $\sigma_{\mathrm{e}}$ is the compression stress and $\varepsilon_{\mathrm{e}}$ is the compression strain.

In Fig 7 it can be seen that the process of the compression of folded core have four stages. The first one is a short stage of elastic deformation. In this stage, the equivalent compression strain $\varepsilon_{\mathrm{e}}$ increases in proportion to the equivalent stress $\sigma_{\mathrm{e}}$. In the second stage, $\sigma_{\mathrm{e}}$ reaches its top value, the cell walls of the folded core buckles, the edges start kinking, and $\sigma_{\mathrm{e}}$ decreases shapely. In the subsequent plateau stage, the cell walls are folding.$\sigma_{\mathrm{e}}$ changes slowly with the increase of $\varepsilon_{\mathrm{e}}, \sigma_{\mathrm{e}}-\varepsilon_{\mathrm{e}}$ curve can be considered appropriately as a horizontal line. Finally, $\sigma_{\mathrm{e}}$ increases again because of the densification of the folded core, it is characterized by a steep slope in the $\sigma_{\mathrm{e}}-\varepsilon_{\mathrm{e}}$ diagram. 


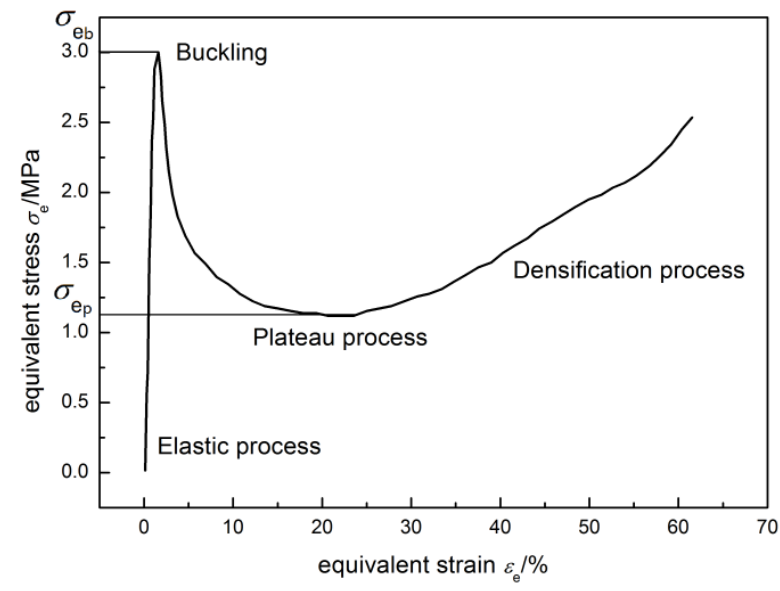

Fig. $7 \sigma_{\mathrm{e}}-\varepsilon_{\mathrm{e}}$ curve of the folded core under compression

In Fig $7, \sigma_{\mathrm{eb}}$-the equivalent buckling stress, $\sigma_{\mathrm{ep}}$-the equivalent plateau stress

Analysis of compute results. In order to prove the accuracy of the calculation results, $\sigma_{\mathrm{e}}-\varepsilon_{\mathrm{e}}$ curve calculated from the FEM model is compared with the experimental result from reference[1]. The finite element model is set up according to the geometric parameters, the loading condition and the material from reference[1]. The comparison can be seen in Fig 8 and Fig.9.

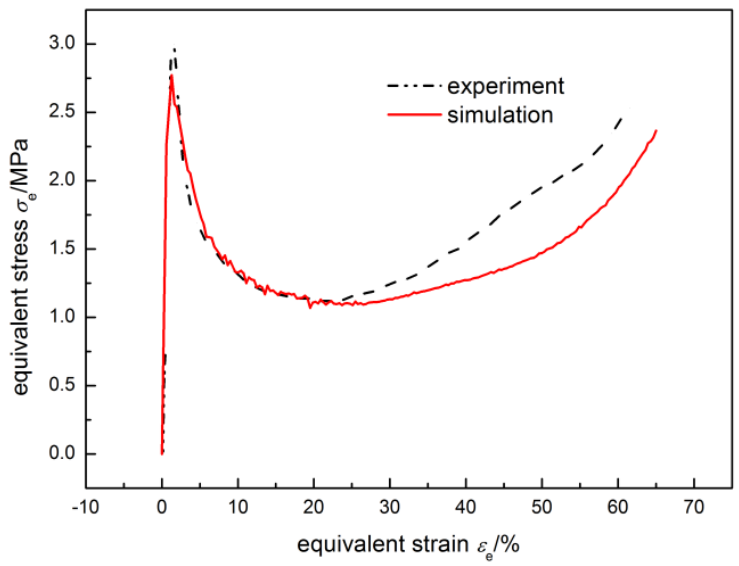

Fig.8 $\sigma_{\mathrm{e}}-\varepsilon_{\mathrm{e}}$ curve

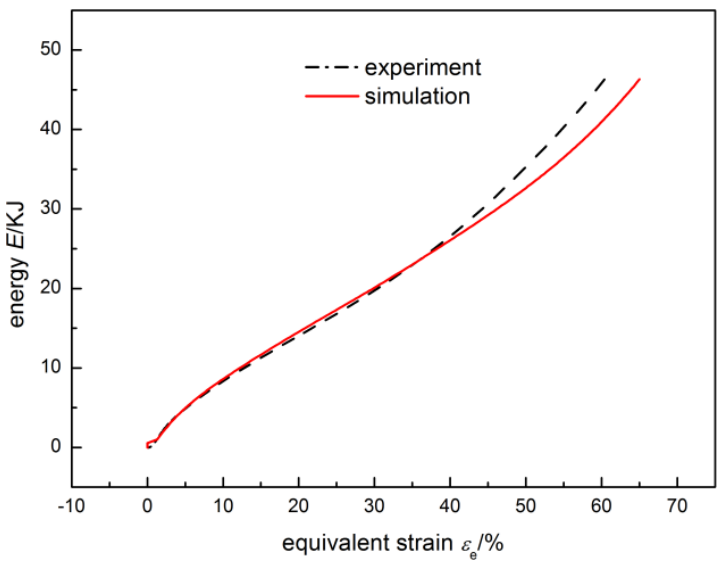

Fig.9 Energy absorbed $-\varepsilon_{\mathrm{e}}$ curve

From Fig 8 it can be seen that our results and the experimental results are very close to each other. The calculated buckling stress $\sigma_{\mathrm{e}}$ is slightly smaller than the experimental value. This is because of the simplification of the aramid paper properties. The calculated $\sigma_{\mathrm{e}}$ after $\varepsilon_{\mathrm{e}}=25 \%$ is also smaller than the experiment result. It is because of that the friction in self-contact of the folded core is ignored. The relationship between energy absorbed in the compression process and $\varepsilon_{\mathrm{e}}$ is presented in Fig 9 . The difference between the two curves is very little.

The deformation states of the folded core is shown in Fig 10.

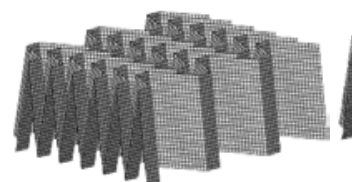

(a) Initial state

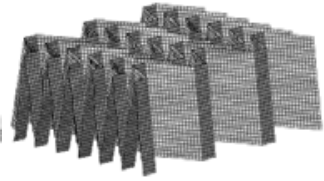

(b) State when

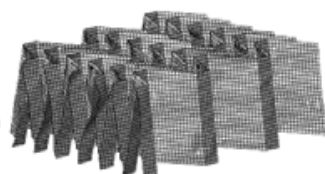

(c)Maximum

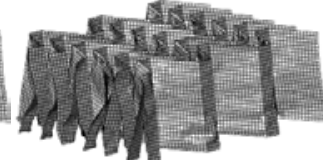

(d) Plateau process

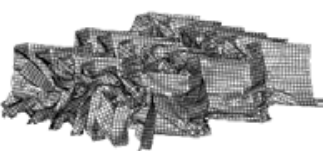

(e) Finish

buckling starts equivalent stress

Fig.10 The deformation states of folded core under compression

Comparison of folded core and honeycomb core. The EAC of one honeycomb core is compared with that of a folded core with the same height, density and material. In reference paper [25] the honeycomb core example is presented, the energy absorbed by honeycomb core can be calculated from Fig.8 in reference paper [25]. The mass of the honeycomb core can be calculated from the geometric parameters and the density of aluminum. And the EAC can be calculated from the energy absorbed and the mass as $5.61 \mathrm{KJ} / \mathrm{Kg}$. According to the height and density of the honeycomb core, the parameters of folded core are set as $A=7 \mathrm{~mm}, B=5 \mathrm{~mm}, \lambda=34^{\circ}, \alpha=11^{\circ}, H=25 \mathrm{~mm}$, the EAC of 
folded core is $14.7 \mathrm{KJ} / \mathrm{Kg}$. The energy absorbing property of folded core is much better than the honeycomb core, which means that folded core sandwich structures are suitable for using as energy absorption structures.

The influence of geometric parameters of folded core on EAC.

Under constant height equal to $20 \mathrm{~mm}$, M-type folded core have four parameters: $A, B, \alpha, \lambda .25$ samples of the folded core are designed as shown in Table 1.

Table 1 The geometric parameters of the folded core samples.

\begin{tabular}{cccccccccccccc}
\hline Number & 1 & 2 & 3 & 4 & 5 & 6 & 7 & 8 & 9 & 10 & 11 & 12 & 13 \\
\hline$A / \mathrm{mm}$ & 12.5 & 12.5 & 12.5 & 12.5 & 12.5 & 12.5 & 12.5 & 12.5 & 12.5 & 12.5 & 12.5 & 12.5 & 12.5 \\
$B / \mathrm{mm}$ & 5 & 5 & 5 & 5 & 5 & 5 & 5 & 5 & 5 & 5 & 5 & 5 & 5 \\
$\alpha{ }^{\circ}$ & 9 & 6 & 7 & 8 & 10 & 11 & 12 & 9 & 9 & 9 & 9 & 9 & 9 \\
$\lambda /^{\circ}$ & 30 & 30 & 30 & 30 & 30 & 30 & 30 & 15 & 20 & 25 & 35 & 40 & 45 \\
\hline Number & 14 & 15 & 16 & 17 & 18 & 19 & 20 & 21 & 22 & 23 & 24 & 25 & \\
\hline$A / m m$ & 4.5 & 6.5 & 8.5 & 10.5 & 14.5 & 16.5 & 12.5 & 12.5 & 12.5 & 12.5 & 12.5 & 12.5 \\
$B / m m$ & 5 & 5 & 5 & 5 & 5 & 5 & 2 & 3 & 4 & 6 & 7 & 8 \\
$\alpha{ }^{\circ}$ & 9 & 9 & 9 & 9 & 9 & 9 & 9 & 9 & 9 & 9 & 9 & 9 \\
$\lambda /^{\circ}$ & 30 & 30 & 30 & 30 & 30 & 30 & 30 & 30 & 30 & 30 & 30 & 30 & \\
\hline
\end{tabular}

The energy absorbing properties of each sample are calculated using FEM and will be analyzed in follow.

The effects of parameter $A$ on EAC. When only $A$ changes and other parameters keep constant, the relation of EAC and $A$ can be got. The relation is shown in Fig 11. And $\sigma_{\mathrm{e}}-\varepsilon_{\mathrm{e}}$ curve of models with different A are shown in Fig 12.

From Fig 11 it can be seen that EAC will increases with the decrease of $A$. From formula (2) it can be seen that when $A$ increases, the mass of the folded core increases. And from Fig 12 it can be seen that when folded core is compressed, $\sigma_{\mathrm{eb}}$ and $\sigma_{\mathrm{ep}}$ slightly decrease with the increase of $A$, which leads to the decrease of the absorbed energy by the folded core. So EAC will decrease with the increase of $A$.

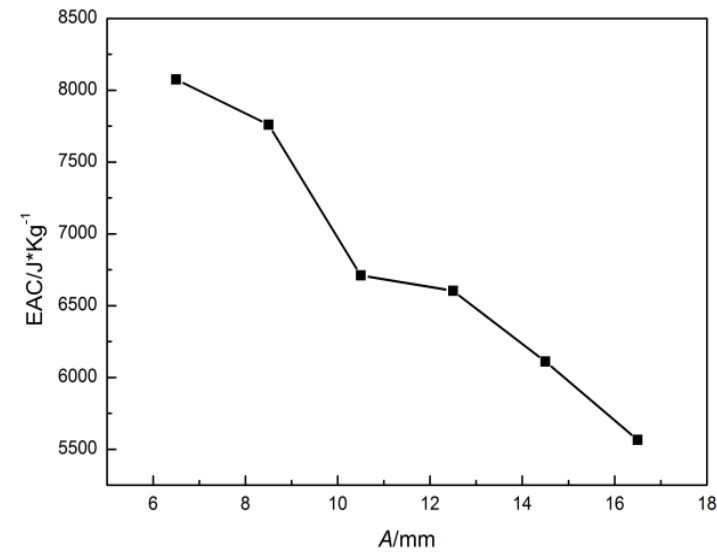

Fig.11 EAC-A curve

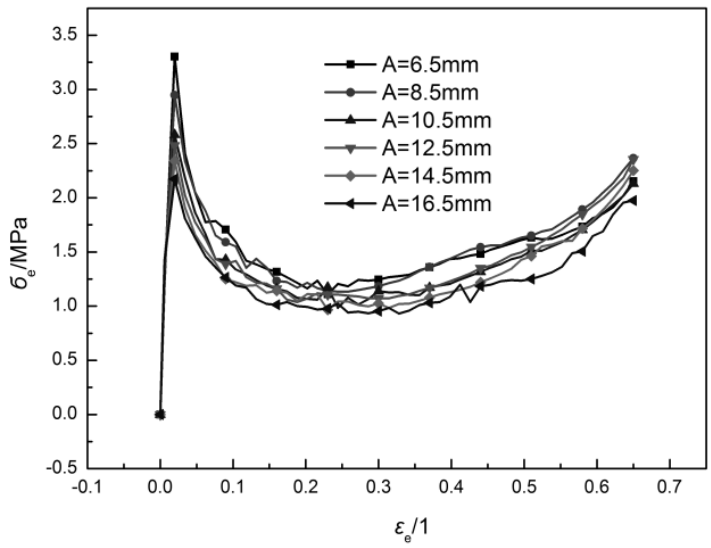

Fig.12 $\sigma_{\mathrm{e}}-\varepsilon_{\mathrm{e}}$ curve corresponding to different $A$

The effects of parameter $B$ on EAC. When $A, \alpha, \lambda$ keep constant and $B$ changes, the relationship of EAC and $B$ can be got and is shown in Fig $13 . \sigma_{\mathrm{e}}-\varepsilon_{\mathrm{e}}$ curves of models with different $B$ are shown in Fig 14.

In Fig 13, when $B$ increases, EAC increases first and then decreases. From formula (2) it can be seen that the mass of the folded core increases with the increase of $B$. And when $B$ is less than $6 \mathrm{~mm}$, the increase of $B$ will lead to the increase of $\sigma_{\text {ep }}$. But when $B$ is bigger than $6 \mathrm{~mm}, \sigma_{\text {ep }}$ decreases when $B$ increases, see in Fig 14. So the energy absorbed first increases and then decrease. Considering that the mass keeps increasing, EAC first increases and then decreases.

In addition, from Fig.14 it also can be seen that $\sigma_{\text {eb }}$ decreases with the increase of $B$, which means that when $B$ increases, the folded core would buckle earlier. 

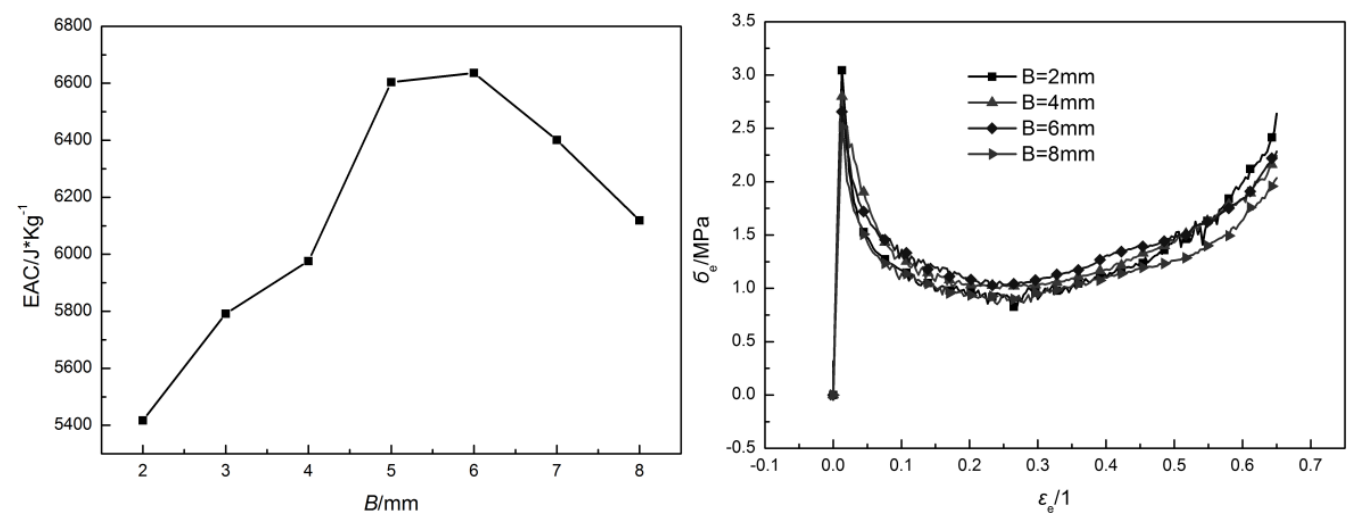

Fig.13 EAC - $B$ curve

Fig. $14 \sigma_{\mathrm{e}}-\varepsilon_{\mathrm{e}}$ curve corresponding to different $B$

When $A$ and $B$ increase, $\sigma_{\text {eb }}$ decreases. The reason is that the parallelogram elements and rectangular elements can all be seen as a thin plate simply supported on four sides. When under compression, the buckling stress can be expressed as:

$$
\sigma_{\mathrm{cr}}=k \frac{\pi^{2} E}{12\left(1-\mu^{2}\right)} \frac{1}{(b / t)^{2}}
$$

In formula (4), $\sigma_{\mathrm{cr}}$-the buckling stress, $t$-the thickness of the thin plate, $b$-the length of the loaded edge. From formula.(4) it can be concluded that the increase of $b$ will lead to the decrease of $\sigma_{\mathrm{cr}}$, which means that $\sigma_{\text {eb }}$ decreases when $A$ or $B$ increases.

The effects of parameter $\boldsymbol{\alpha}$ on EAC. When $\alpha$ changes while $A, B$ and $\lambda$ keep constant, the relation of EAC and $\alpha$ can be got. It is shown in Fig 15.

From Fig 15 it can be seen that, EAC decreases with the increase of $\alpha$. From formula (2) it can be seen that when $\alpha$ increases, the mass of the folded core increases. And in Fig 16 it can be seen that when $\alpha$ increases, $\sigma_{\text {eb }}$ and $\sigma_{\text {ep }}$ will decrease, and the start of the densification process will be delayed. So EAC decreases with the increase of $\alpha$.

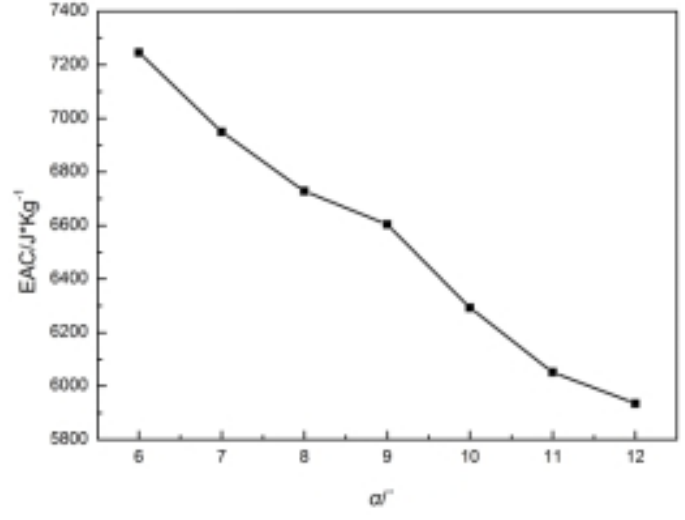

Fig.15 EAC- $\alpha$ curve

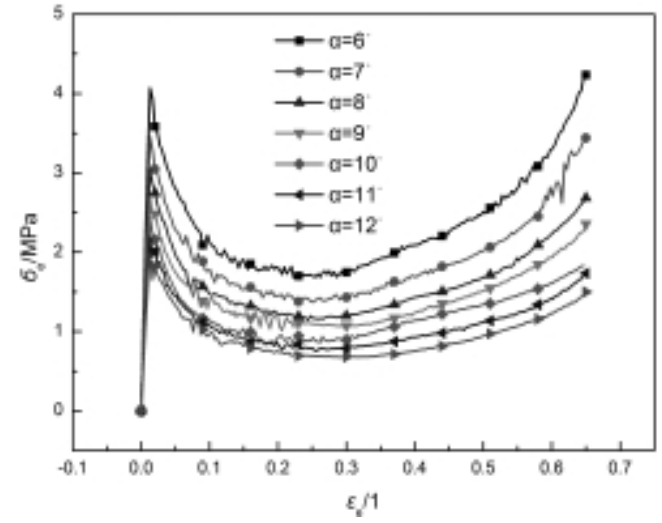

Fig.16 $\sigma_{\mathrm{e}}-\varepsilon_{\mathrm{e}}$ curve corresponding to different $\alpha$

The effects of parameter $\lambda$ on EAC. When $A, B, \alpha$ keep constant and $\lambda$ changes, the relation of EAC and $\lambda$ can be got. The relation is shown in Fig 17.

From Fig 17 it can be seen that EAC decreases with the increase of $\lambda$. From formula (2) it can be seen that the mass of the folded core do not related with $\lambda$. And in Fig $18, \sigma_{\mathrm{eb}}$ and $\sigma_{\text {ep }}$ decrease with the growth of $\lambda$, So when $\lambda$ increases, the energy absorbed decreases. 


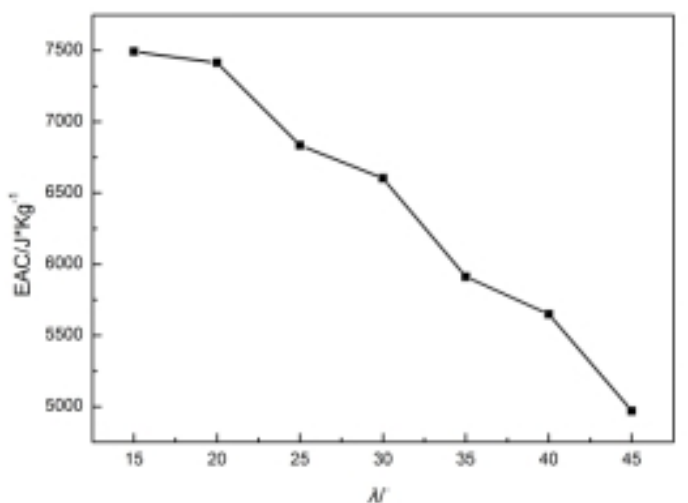

Fig.17 EAC- $\lambda$ curve

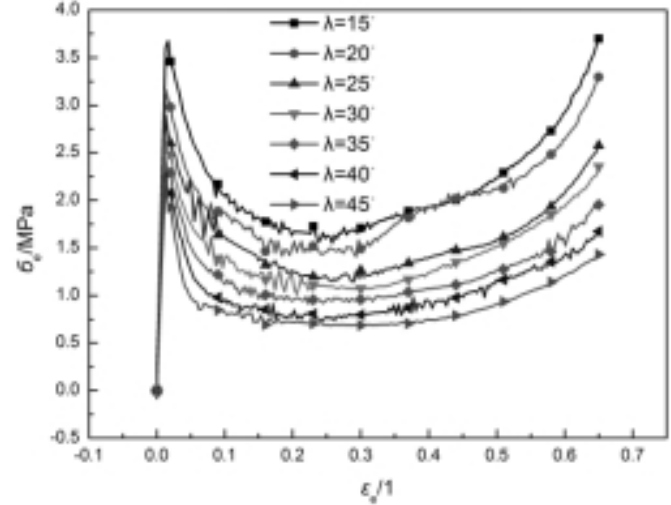

Fig.18 $\sigma_{\mathrm{e}}-\varepsilon_{\mathrm{e}}$ curve corresponding to different $\lambda$

\section{Summary}

(1) Explicit dynamic analysis is an appropriate way to model the compression process of M-type folded core. The analysis result is compared with the experiment result from CELPACT project, they match well.

(2) One folded core which have same height, same density and same material with a honeycomb core is analyzed. Folded core can absorb much more energy than honeycomb core.

(3) The influences of geometric parameters of folded core on EAC are analyzed. With the increasing of $A, \alpha$ and $\lambda$, EAC decreases. With the increase of $B$, EAC first increases and then decreases.

\section{References}

[1] Johnson A, Novel hybrid structural core sandwich materials for aircraft applications. 11th Euro-Japanese Symposium on Composite Materials.2008.

[2] Wang Zhijin, Xu Qinghua, Experimental research on soundproof characteristic for the sandwich plates with folded core. JOURNAL OF VIBRATION ENGINEERING. 19(2006) 65-69.

[3] Wang Zhijin, Xu Qinghua, Research on RCS of Plates with Folded Structure Made of Aluminum Foil. ACTA AERONAUTICA ET ASTRONAUTICA SINICA. 29(2008) 1213-1217.

[4] Wang Zhijin, Zhang Hui, Xu Qinghua, Equivalent Thermal Conductivity Coefficient for Sandwich Plates with Folded Core. Journal of Nanjing University of Aeronautics \& Astronautics. 40(2008) 507-512.

[5] Xu Qinghua, Research on Thermodynamic characteristics for the Metallic Sandwich plates with folded core. M.A. thesis, Nanjing University of Aeronautics and Astronautics, 2006

[6] Wang Zhijin, Khaliulin V I, Geometry Design Method of Folded Structure. Journal of Nanjing University of Aeronautics \& Astronautics. 34(2002): 6-11.

[7] Wang Zhijin, Xu Qinghua, Meso-Mechanical Model of Elastic Constants of M-Type Folded Core. Journal of Nanjing University of Aeronautics \& Astronautics. 36(2004): 449-453.

[8] Shao Changlin, Wang Jianbing, Zhao Houliang, Research of Folder-filler Structure's Electromagnetic Shield Efficiency. Journal of Shandong Institute of Building Materials. 15(2001) 19-22.

[9] Shao Changlin, Wang Jianbing, Experimental Research of Folder-filler Structure's Soundproof Characteristics. Journal of Shandong Institute of Building Materials. 13(1999): 302-304.

[10]Heimbs S, Middendorf P, Hampf C, et al. Aircraft sandwich structures with folded core under impact load. Proc. of the 8th International Conference on Sandwich Structures. (2008) 369-380. 
[11]Heimbs S, Middendorf P, Kilchert S, et al. Experimental and numerical analysis of composite folded sandwich core structures under compression. Applied Composite Materials.14(2007) 363-377.

[12]Heimbs S, Middendorf P, Kilchert S, et al. Numerical simulation of advanced folded core materials for structural sandwich applications. 1st CEAS European Air and Space Conference. (2007) 2889-2895.

[13] Heimbs S, Virtual testing of sandwich core structures using dynamic finite element simulations. Computational Materials Science. 45(2009) 205-216.

[14]Heimbs S, Mehrens T, Middendorf P, et al. Numerical determination of the nonlinear effective mechanical properties of folded core structures for aircraft sandwich panels. 6th European LS-Dyna users conference. 5(2007) 181-5.190.

[15]Heimbs S, Cichosz J, Kilchert S, et al. Sandwich panels with cellular cores made of folded composite material: mechanical behavior and impact performance. 17th international conference on composite materials (ICCM-17).(2009).

[16]Fischer S, Heimbs S, Kilchert S, et al. Sandwich structures with folded core: manufacturing and mechanical behavior. SAMPE Europe international conference.(2009) 256-263.

[17]Heimbs S, Cichosz J, Klaus M, et al. Sandwich structures with textile-reinforced composite fold cores under impact loads. Composite Structures. 92(2010) 1485-1497.

[18]Klaus M, Reimerdes H G, Gupta N K. Experimental and numerical investigations of residual strength after impact of sandwich panels. Journal of Impact Engineering 44(2012) 50-58.

[19]Fischer S, Drechsler K, Kilchert S, et al. Mechanical tests for foldcore base material properties. Composites Part A: Applied Science and Manufacturing. 40(2009) 1941-1952.

[20]Baranger E, Guidault P A, Cluzel C. Numerical modeling of the geometrical defects of an origami-like sandwich core. Composite Structures. 93(2011) 2504-2510.

[21]Baranger E, Cluzel C, Guidault P A. Modeling of the behavior of aramid folded cores up to global crushing. Strain. 47(2011) 170-178.

[22]Hähnel F, Wolf K, Hauffe A, et al. Wedge-shaped folded sandwich cores for aircraft applications: from design and manufacturing process to experimental structure validation. CEAS Aeronautical Journal. 2(2011) 203-212.

[23]Aminanda Y, Castanie B, Barrau J J, et al. Experimental analysis and modeling of the crushing of honeycomb cores. Applied Composite Materials. 12(2005) 213-227.

[24]ABAQUS: User's Manual.(1997)

[25]Hong S T, Pan J, Tyan T, et al. Quasi-static crush behavior of aluminum honeycomb specimens under compression dominant combined loads. International Journal of Plasticity. 22(2006) 73-109. 